\title{
Probing Deep Tissues with Laser-Induced Thermotherapy Using Near Infrared Light
}

Received: date / Accepted: date

\begin{abstract}
Optically tunable gold nanoparticles have been widely used in research with near-infrared light as a means to enhance laser-induced thermal therapy since it capitalises on nanoparticles' plasmonic heating properties. There have been published several studies on numerical models replicating this therapy in such conditions. However, there are several limitations on some of the models which can render the model unfaithful to therapy simulations. In this paper, two techniques of simulating laser induced thermal therapy with a high absorbing localised region of interest inside a phantom are compared. To validate these models we conducted an experiment of an agar-agar phantom with an inclusion reproducing it with both models. The phantom was optically characterized by absorption and total attenuation. The first model is based on the macro perspective solution of the radiative transfer equation given by the diffusion equation, which is then coupled with the Pennes bioheat equation to obtain temperature. The second is a Monte Carlo model that considers a stochastic solution of the same equation and is also considered as input to the Pennes bioheat transfer equation which is then computed. The Monte Carlo is in good agreement with the experimental data having an average percentage difference of $4.5 \%$ and a correlation factor of 0.98 , while the diffusion method comparison with experimental data is $61 \%$ and 0.95 respectively. The optical characterisation of the phantom and its inclusion were also validated indirectly since the Monte Carlo, which used those parameters, was also validated. While knowing the temperature in all points inside a body during photothermal therapy is important, one has to be mindful of the model which fit the conditions and properties. There are several reasons to justify the
\end{abstract}

discrepancy of the diffusion method: low scattering conditions, absorption and reduced scattering are comparable. The error bars that are normally associated when characterizing an optical phantom can justify also a part of that uncertainty. For low size tumours in depth, one may have to increase the light dosage in photothermal therapies to have a more effective treatment.

Keywords Phototherapy · Photothermal therapy · Monte Carlo · Diffusion Approximation · Near Infrared Light

\section{Introduction}

Breast cancer remains the leading cancer killer in middle aged women around the world [15]. Despite recent advances in therapy and diagnosis, cancer continues to be a difficult disease to treat. Depending on its location, stage or whether it became resistant to the ongoing treatment, the therapies can also change. Therefore, it is imperative that the resources the oncologist has to tackle this disease, be numerous and diverse. In this work, we study near infrared photothermal therapy (PTT), which allow non-ionizing radiation to diffuse inside tissue and cause low temperature elevation $[11,14]$. When compared to the most conventional therapies like surgery and chemotherapy, it is non-invasive and produces minimal damage to healthy tissue. A fraction of photon energy is absorbed and converted into heat, depending on tissue properties, which can destroy malignant tissue through processes like coagulation or hyperthermia if the achieved temperature and time of exposure at that temperature is sufficient [2]. A limiting factor in this process is large optical scattering in biological tissue which imposes restrictions on how deep one can heat a predetermined region of interest. With 
the advent of gold nanoparticles (GNP) in medicine, there is a promising opportunity to bypass such limitations.

Gold nanoparticles have tunable high absorption properties in the NIR regions that allow local higher absorbed energy. Several authors $[25,5,4,19]$, report a temperature change at up to $3 \mathrm{~cm}$ depth when using these particles while no temperature change was observed without them. This increase in temperature caused by exogenous contrast agents changes the rate of deposited heat which in turn is influenced by irradiation conditions and tissue properties that ultimately influence therapy's success [24].

A myriad of cell damaging effects can be observed starting from $\sim 39^{\circ} \mathrm{C}$ where protein denaturation happens. Depending on the time of exposure these effects can do reversible or irreversible damage to cells[20,14, 11]. Hence, a precise temperature control is crucial since it is impossible to have probes to measure the temperature in all space. Consequently, it is important to have models that can mimic the illumination conditions and tissue properties, and allow an accurate temperature representation.

Among the many light models already developed two stand out for being the most used in the community: diffusion model and Monte Carlo. Monte Carlo simulations give a stochastic representation of the solution to the radiative transfer equation (RTE), which is the equation that governs how light propagates inside biological tissue [26]. The diffusion equation can be obtained from the RTE considering some of its parameters as first-order spherical harmonic expansions which allows only isotropic and first-order anisotropic terms. In practice, this imposes restrictions on the conditions in which this theory is valid, i.e. on tissues with low absorption and high scattering properties, among others [24].

The studies that are published using nanoparticles can be separated into two categories. There are some approaches when using gold nanoparticles in the literature that consider an homogeneous mixture of a solution and gold nanoparticles and replicate the temperature or irradiance with simulations [23,25]. Other approaches consider two layers of tissue stacked with and without the presence of gold nanoparticles $[4,5]$. Depending on the number of gold nanoparticles used, the optical properties of the tissue vary significantly to a point where absorption is comparable to scattering [18], which might render the diffusion theory ineffective. In this work, we will address this problem using an agar phantom with an inclusion that mimics this condition. First, it is presented the conventional method of measuring some of the phantoms' optical properties.
After, an experiment is conducted in which the phantom is irradiated and the temperature is measured with a thermocouple inside the phantom.

The numerical methods consider the light distribution using diffusion theory and Monte Carlo, which is considered the gold standard to model light [26]. To model the heat transfer mechanism we choose the Pennes bioheat equation [17] that reproduces well the temperature inside a phantom [24].

In this paper, we present a study with a nanoparticle based inclusion in a phantom far from its surface. To the best of the author's knowledge this study is not presented elsewhere and is important; the nanoparticles aggregate around a tumour of finite size and the other studies present homogeneous mediums with nanoparticles[5]. We also characterize the optical properties of the mediums considered and the irradiation experiment.

\section{Mathematical formulation}

The models of optical and thermal propagations of light and heat through the tissue are presented in this section. In the first part, we consider the diffusion theory and Monte Carlo simulations to solve the radiative transfer equation, while the Pennes bioheat transfer equation is considered to model the heat propagation mechanism.

The diffusion equation with a constant wave source at its border is defined as [1]

$-\nabla \cdot \kappa(\mathbf{r}) \nabla \phi(\mathbf{r})+\mu_{a}(\mathbf{r}) \phi(\mathbf{r})=0, \mathbf{r} \in \Omega$,

where $\kappa=1 /\left(3\left(\mu_{s}^{\prime}+\mu_{a}\right)\right)$ is the diffusion coefficient, $\mu_{a}$ the absorption coefficient, $\mu_{s}^{\prime}$ is the reduced scattering coefficient, $\phi$ the fluence rate, $\Omega$ its domain and position vector $\mathbf{r}$. The fluence rate boundary conditions between phantom and exterior are defined by

$\phi(\mathbf{m})+2 \xi(c) \kappa(\mathbf{m}) \frac{\partial \phi(\mathbf{m})}{\partial \nu}=q(\mathbf{m}), \mathbf{m} \in \partial \Omega$,

where the term $\xi(c)$ represents the refractive boundary mismatch between different regions, $\partial \nu$ represents the outward boundary normal, $q$ is the source distribution at the boundary $\partial \Omega$, and $\mathbf{m}$ the position vector restricted to $\partial \Omega$. This model is derived under two premises, it is valid in high diffusive versus absorbing tissues and it is not valid near source points, where the gradient of fluence rate is not linear [24].

In a Monte Carlo simulation, each photon is simulated individually with a predetermined starting position, direction and interaction mechanisms. In the near 
infrared region, light either scatter or gets absorbed inside an homogeneous tissue. The scattering phase function of the radiative transfer equation in soft biological tissues can be modelled by the Henyey-Greenstein phase function [24]. At the boundaries, refraction and reflection are considered with Fresnel and Snell equations [7].

The laser induced heating source term is described by $Q(\mathbf{r}, t) \equiv \mu_{a}(\mathbf{r}) \times \phi(\mathbf{r}, t)[24]$. Since this term is external to the heat generated in the body, we add it to the Pennes bioheat transfer equation as follows

$$
\begin{aligned}
\rho c \frac{\partial T(\mathbf{r}, t)}{\partial t}= & \nabla \cdot(k(\mathbf{r}) \nabla T(\mathbf{r}, t))+Q_{m}(\mathbf{r})+Q(\mathbf{r}, t) \\
& +\omega_{b} \rho_{b} c_{b}\left(T(\mathbf{r}, t)-T_{a}\right), \mathbf{r} \in \Omega
\end{aligned}
$$

where $\rho$ is the tissue density, $c$ is the specific heat, $T(\mathbf{r}, t)$ the temperature, $k(\mathbf{r})$ the thermal conductivity, $Q_{m}$ metabolic heat rate, $\omega_{b}$ rate of blood perfusion, $\rho_{b}$ density of blood, $c_{b}$ blood's specific heat and $T_{a}$ body's temperature.

The convection heat transfer mechanism is also considered and is determined by Newton's law:

$Q_{c}=h\left(T_{\infty}-T(\mathbf{m}, t)\right), \mathbf{m} \in \partial \Omega$,

where $h$ is the convective heat transfer coefficient and $T_{\infty}$ is the room temperature. Radiation, thermal evaporation and/or phase change of tissue during the heat transfer process are not considered in the simulations.

\section{Materials and Methods}

An agar-agar phantom was produced in two steps. First, a mixture of $1.0 \%$ agarose powder (Agar-Agar, Vahiné, France) in distilled water. The water was heated along with the agarose powder and mixed (at a mixing temperature of $\approx 90^{\circ} \mathrm{C}$ ) three times to ensure an homogeneous optical density of the gel. It was then let cool off inside a $60 \times 60 \times 60 \mathrm{~mm}^{3}$ cube using a cylinder of 15.5 $\mathrm{mm}$ in diameter to create a $5 \mathrm{~mm}$ deep hole on top of the phantom. Once it cooled down to ambient temperature, the hole created by the cylinder was filled with two equal parts of agar and gold nanoparticles prepared according to $[22]$. The two were mixed at $35^{\circ} \mathrm{C}$ since the gold nanoparticles had a bio-coating in them. This proportion was used to ensure the gold nanoparticles were in a fixed place, but also to have a good balance between scattering and absorption. A $4 \mathrm{~mm}$ layer of agar-agar solution was added to the phantom. The final phantom is depicted in figure 1.
Fig. 1 Gel phantom setup. The laser beam entry position and direction is depicted in red. The main component (agar) is made of $1 \%$ agar and water, while the smaller component (GNP) is made of $1 \%$ agar, $10 \%$ GNP solution and $89 \%$ water.

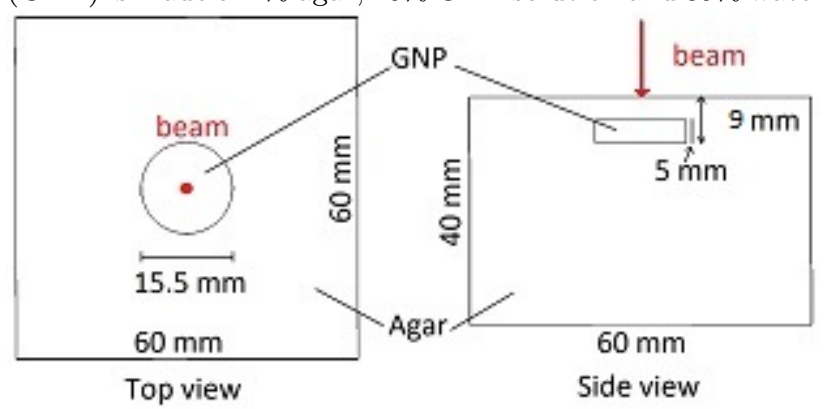

Determination of Materials' Properties

The optical properties of the gel and inclusion were measured using an integrating sphere (International Light, INS 250), a laser diode source (Roithner, RLTMDL-808-5W-5) $808 \mathrm{~nm}$ wavelength and a spectrometer (Avaspec 2048, Avantes). The measured coefficients were the absorption coefficient and the total attenuation coefficient. Other optical properties such as anisotropy and refractive index were taken from literature $[5,10]$.

Similarly to the methods described in [16], the absorption coefficient $\left(\mu_{a}\right)$ was measured placing the cuvette with a sample of agar or nanoparticles and agar in the integrating sphere's centre, and compared with a cuvette with distilled water. Coupled with the integrating sphere was the spectrometer whose signal was then deconvoluted from the laser wavelength distribution peaking at $808 \mathrm{~nm}$. The absorption coefficient was determined using the equation $\mu_{a}=\frac{I_{0}-I}{I_{0}}$, where $I_{0}$ is the measurement with distilled water, and $I$ the signal measured with the sample solution.

To measure the attenuation coefficient $\left(\mu_{t}\right)$ a procedure similar to the one described in [16] was followed. Additionally, a 1mm-pinhole was placed in front of the detector and both were positioned $40 \mathrm{~cm}$ away from the sample. This procedure is reported to limit multiple scattering events to some extent $[12,13]$. The BeerLambert equation was used to determine the attenuation coefficient, $\mu_{t}=-\log \frac{I}{I_{0}}$, where $I$ is the measured signal of the cuvette with the sample and $I_{0}$ the measured signal of the cuvette with distilled water. Each measurement was repeated 5 times to ensure consistency. The scattering coefficient was determined indirectly by the equation $\mu_{s}=\mu_{t}-\mu_{a}$. Table 1 shows the results of the two mixtures used in the phantom. 


\begin{tabular}{|c|c|c|c|}
\hline Sample & $\mu_{a}\left[\mathrm{~mm}^{-1}\right]$ & $\mu_{t}\left[\mathrm{~mm}^{-1}\right]$ & $\mu_{s}\left[\mathrm{~mm}^{-1}\right]$ \\
\hline Gel & 0.002 & 0.178 & 0.176 \\
\hline GNP & 0.031 & 0.320 & 0.289 \\
\hline
\end{tabular}

Table 1 Measured optical properties of the agar gel phantom and a mixture of gold nanoparticles (GNP) and agar gel.

\section{Experimental setup}

The phantom was irradiated in the top surface of the box with a continuous wave diode laser of $1.1 \mathrm{~W}$ power and a full width at half maximum of $7 \mathrm{~mm}$, as represented in figure 1.

Temperatures were registered using a type $\mathrm{K}$ thermocouple (Labfacility, Z2-T-1M) and a thermocouple converter (Seneca K109TC) connected to a LabVIEW ${ }^{\circledR}$ interface and with a tunable data acquisition rate of $1 \mathrm{~s}$. The thermocouple was placed on top of the cylinder, at $4 \mathrm{~mm}$ depth. The constant wave laser was on for $706 \mathrm{~s}$, then turned off. The radiation absorbed by thermocouple was taken into account and was subtracted from experimental results as suggested in [3]. The ambient temperature was measured at $22.9{ }^{\circ} \mathrm{C}$ and the temperature measured in the thermocouple the instant before the laser irradiation phase was $23.88^{\circ} \mathrm{C}$, which is considered the reference temperature when determining temperature change.

\section{Simulations}

Several software applications were used to compute the solution to the temperature determination. Along with the commercial software COMSOL Multiphysics ${ }^{\circledR}$ and MATLAB ${ }^{\circledR}$, which were used to perform the data analysis. Iso2mesh [8] and GMSH [9] were used to build and characterize the meshed phantom. This mesh was used as an input in both TOAST $++[21]$ and the Monte Carlo software Mesh-based Monte Carlo [6]. Both of these codes are considered to be the state of the art in their own domain to determine the irradiance within biological tissue. One only needs to provide the laser parameters and the tissue's optical properties since the appropriate functions are already built-in. All codes were run in a 8x Intel Core i7 $4790(4.0 \mathrm{GHz}) \mathrm{CPU}$ with 4x 4GB DDR3 1600MHz.

The diffusion approximation software provides the selection of a gaussian profiled source with $\sigma=3.07$ $\mathrm{mm}$, modelled as a Neumann source which considers it as a diffuse boundary current. The same laser profile and the standard deviation were selected in the Monte Carlo simulator. 10 million events were simulated. The output fluence rate of both programs was then used as $3 \mathrm{D}$ heat source in COMSOL.
The temperature for both cases was computed by COMSOL Multiphysics ${ }^{\circledR}$. The top surface transferred convective heat using the Newton convection heat transfer equation with the respective coefficient at $5 \mathrm{~W} \mathrm{~K}^{-1}$ $\mathrm{m}^{-2}[24]$ while the other surfaces were considered to be in thermal isolation. Although this consideration could be considered erroneous, its effect is considerable only after the laser-on phase when the phantom is cooling down. The blood density and the metabolic heat coefficient of the Pennes bioheat equation were set to zero. This consideration converts the Pennes bioheat equation into the classical heat transfer equation. The density, heat capacity and thermal conductivity coefficients of water were considered in this test.

The used optical parameters for the phantom are the following: absorption coefficient: $\mu_{a}: 0.002 \mathrm{~mm}^{-1}$, reduced scattering coefficient: $\mu_{s}^{\prime}=\mu_{s}(1-g)=0.0176$ $\mathrm{mm}^{-1}$ an anisotropy $(g)$ of 0.9 is assumed [5], refractive index $=1.33[10]$. The inclusion's optical properties differ from the previous ones on the absorption coefficient, which is $\mu_{a}=0.031 \mathrm{~mm}^{-1}$ and reduced scattering coefficient $\mu_{s}^{\prime}=0.029 \mathrm{~mm}^{-1}$, while the others are the same. Inside the inclusion the absorption coefficient and the reduced scattering coefficient are comparable and can make the diffusion approximation ineffective.

\section{Results and Discussion}

To check that both models work within reasonable agreement a simulation was conducted. Consider a case in which both Monte Carlo and the diffusion approximation are effective; an homogeneous $2 \mathrm{~cm}$ wide cube with the following optical properties: $\mu_{a}=0.002 \mathrm{~mm}^{-1}$, $\mu_{s}^{\prime}=1.22 \mathrm{~mm}^{-1} ; g=0.9$ and refractive index of 1.33 . A continuous wave gaussian near infrared laser beam of power $=1.5 \mathrm{~W}$ and $\mathrm{FWHM}=5 \mathrm{~mm}$ aims at one of the cube's side at the centre for 180 s. Figure 2 shows a comparison between temperature increase of both simulators $1 \mathrm{~mm}$ deep of the irradiated side. One can observe the diffusion approximation is almost $1 \mathrm{~K}$ higher at $180 \mathrm{~s}$ between simulated temperatures which is caused by the different approaches of dealing with scattering anisotropy by the two models.

Figure 3 presents the comparison between experiment and simulation results given from Monte Carlo and diffusion theory. Both models are in good agreement with the experiment measurements. There are some variations in the measured temperature along time which is explained by non-linear photon absorption and heat diffusion from the thermocouple probe unto its surroundings. The instant the irradiation phase stopped this behaviour was not observed. These variations decrease in amplitude with smaller thermocouple probes. 
Fig. 2 Temperature increase comparison between Monte Carlo (blue) and the diffusion theory (green) simulators as a function of time, at a point located $1 \mathrm{~mm}$ below the irradiated spot.

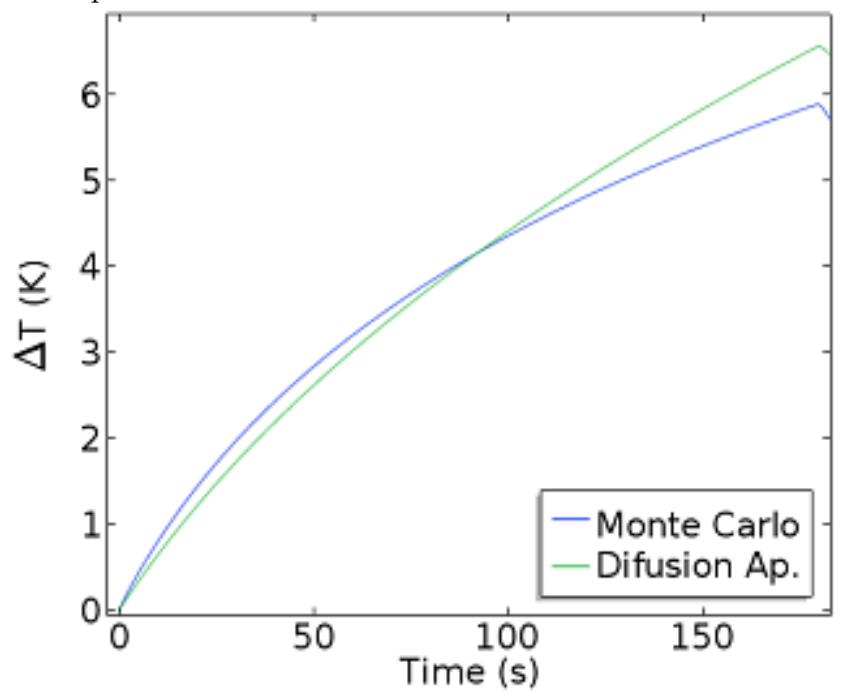

The average percentage difference between the Monte Carlo model is $4.5 \%$ and for the diffusion approximation $61 \%$, while the correlation coefficient was of 0.98 and 0.95 , respectively.

These results can be compared to e.g., Elliot et al. [5] where this problem is explored in a similar experiment. Joined in a single phantom cylinder are two smaller cylinders with different optical properties and lengths. The diffusion approximation was used to model the experiment. The physical parameters for the finite element calculations were adjusted within the experimental error $( \pm 10 \%)$ to optimize results. The average percentage difference and correlation coefficient were $4.5 \%$ and 0.99 respectively, for the similar conditions as this experiment. Changing the physical parameters in the diffusion approximation model in this study by $20 \%$, decreases the average percentage difference from $61 \%$ to $23 \%$. Although this variation is far from the $4.5 \%$ reported in [5] this result highlights the model sensitivity on these parameters.

Figure 4 shows the temperature increase in depth along the axis that is aligned with the laser beam axis the second before the laser was shut down. The distribution of both models is slightly different. Using more thermocouple probes would allow a more comprehensive study of this distribution. When comparing it with similar temperature change distributions in other studies, such as $[5,4]$, increases in temperature greater than $10 \mathrm{~K}$ can be observed. This result suggests that the size of the inclusion with nanoparticles is a relevant component to temperature increase, along with irradiance as
Fig. 3 Comparison of simulation and experimentally measured increase in temperature, $4 \mathrm{~mm}$ below surface. It shows temperature change as a function of time. Both models are shown in green and red and are compared with the experimental results shown in blue.

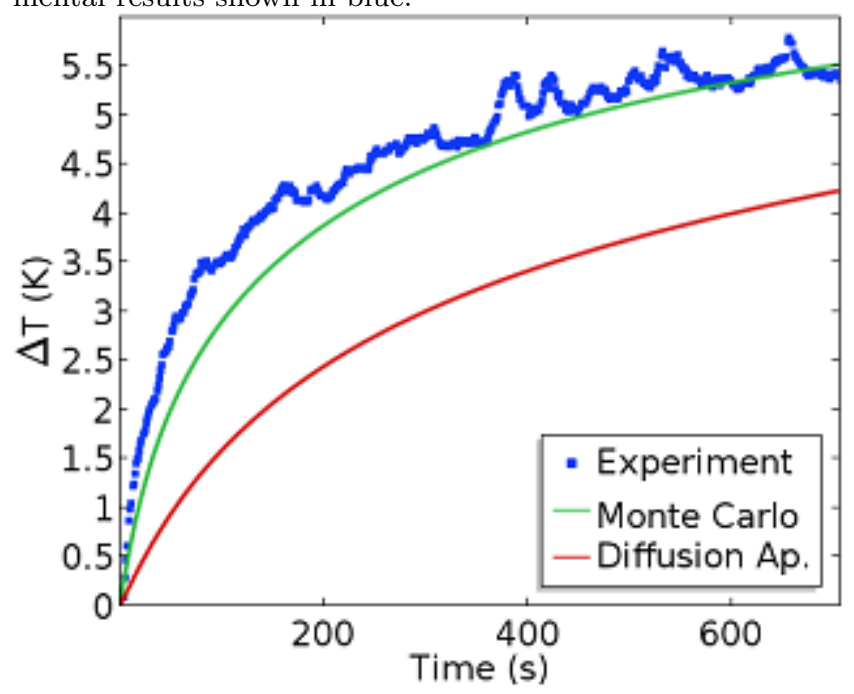

well as the inclusions' optical properties, density and location inside tissue.

Fig. 4 Temperature increase at $t=706 \mathrm{~s}$ right before the laser was turned off. The $\mathrm{x}$-axis represents an axis that is aligned with the laser beam axis, which is located at the centre of two faces of the cube. The Monte Carlo simulation and diffusion approximation results are presented in green and red, respectively. The experimental point is shown in blue.

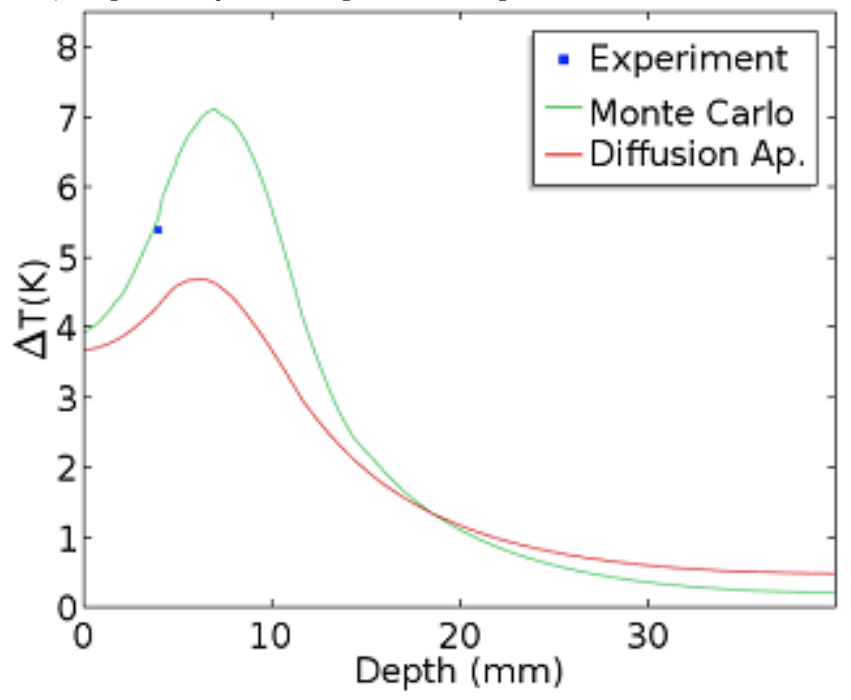




\section{Conclusions}

It is of crucial importance to know the temperature in every point inside the tissue when performing photothermal therapy to better control the light dosage delivered. We present two ways of simulating laser induced thermal therapy in a specific experimental setup with a high absorbing region of interest located at 4 $\mathrm{mm}$ of depth, and compared to experimental data. The two models are in reasonable agreement with the experiment results. The size of inclusion affects the maximum temperature one can achieve, as well as concentration and depth.

Conflict of interests The authors declare that they have no conflict of interest.

Role of funding source This work was partially supported by national funding by the Portuguese FCT - Fundação para a Ciência e Tecnologia through the projects PD/BD/105920/2014, UID/FIS/04559/2013 (LIBPhys) and UID/BIO/00645/2013.

Ethics approval Not applicable.

\section{References}

1. Arridge, S.R.: Optical tomography in medical imaging. Inverse Problems 15(2), R41 (1999). DOI 10.1088/0266$5611 / 15 / 2 / 022$

2. Brunetaud, J.M., Mordon, S., Maunoury, V., Beacco, C.: Non-PDT Uses of lasers in oncology. Lasers In Medical Science 10(1), 3-8 (1995). DOI 10.1007/BF02133156

3. Cain, C.P., Welch, A.J.: Thin-Film Temperature Sensors for Biological Measurements. IEEE Transactions on Biomedical Engineering BME-21(5), 421-423 (1974). DOI 10.1109/TBME.1974.324415

4. Cheong, S.S.K., Krishnan, S., Cho, S.H.: Modeling of Plasmonic Heating From Individual Gold Nanoshells for Near-Infrared Laser-Induced Thermal Therapy. Medical Physics 36(6), 4664-4671 (2008). DOI 10.1118/1.2961677

5. Elliott, A.M., Stafford, R.J., Schwartz, J., Wang, J., Shetty, A.M., Bourgoyne, C., O'Neal, P., Hazle, J.D.: Laser-induced thermal response and characterization of nanoparticles for cancer treatment using magnetic resonance thermal imaging. Medical Physics 34(7), 31023108 (2007). DOI 10.1118/1.2733801

6. Fang, Q.: Mesh-based Monte Carlo method using fast ray-tracing in Plücker coordinates. Biomedical optics express 1(1), 165-175 (2010). DOI 10.1364/BOE.1.000165

7. Fang, Q., Boas, D.a.: Monte Carlo simulation of photon migration in 3D turbid media accelerated by graphics processing units. Optics express 17(22), 20178-20190 (2009). DOI 10.1364/OE.17.020178

8. Fang, Q., Boas, D.A.: Tetrahedral mesh generation from volumetric binary and grayscale images. Proceedings - 2009 IEEE International Symposium on Biomedical
Imaging: From Nano to Macro, ISBI 2009 pp. 1142-1145 (2009). DOI 10.1109/ISBI.2009.5193259

9. Geuzaine, C., Remacle, J.F.: Gmsh: a three-dimensional finite element mesh generator with built-in pre-and postprocessing facilities. International Journal for Numerical Methods in Engineering 79(11) 0, 1309-1331 (2009). DOI 10.1002/nme.2579

10. Jacques, S.L.: Optical Properties of Biological Tissues: A Review. Physics in Medicine and Biology 58(11), R37 (2013). DOI 10.1088/0031-9155/58/11/R37

11. Jaque, D., Martínez Maestro, L., del Rosal, B., HaroGonzalez, P., Benayas, A., Plaza, J.L., Martín Rodríguez, E., García Solé, J.: Nanoparticles for photothermal therapies. Nanoscale 6(16), 9494-9530 (2014). DOI $10.1039 / \mathrm{c} 4 \mathrm{nr} 00708 \mathrm{e}$

12. Kim, A., Wilson, B.C.: Measurement of ex vivo and in vivo tissue optical properties: Methods and theories. In: Optical-Thermal Response of Laser-Irradiated Tissue, pp. 267-319. Springer Netherlands, Dordrecht (2010). DOI 10.1007/978-90-481-8831-4_8

13. Marchesini, R., Bertoni, A., Andreola, S., Melloni, E., Sichirollo, a.E.: Extinction and absorption coefficients and scattering phase functions of human tissues in vitro. Applied optics 28(12), 231824 (1989). DOI 10.1364/AO.28.002318. URL http://www.ncbi.nlm.nih.gov/pubmed/20555518

14. Niemz, M.H.: Laser-Tissue Interactions. Springer Berlin Heidelberg, Berlin, Heidelberg (1996). DOI $10.1007 / 978-3-662-03193-3 . \quad$ URL http://link.springer.com/10.1007/978-3-662-03193-3

15. Organization, W.H.: Women's health (2013). URL http://www.who.int/mediacentre/factsheets/fs334/en/

16. Patterson, M.S., Wilson, B.C., Wyman, D.R.: The propagation of optical radiation in tissue. II: Optical properties of tissues and resulting fluence distributions. Lasers in Medical Science 6(4), 379-390 (1991). DOI 10.1007/BF02042460

17. Pennes, H.H.: Analysis of tissue and arterial blood temperatures in the resting human forearm. 1948. Journal of applied physiology (Bethesda, Md. : 1985) 85, 5-34 (1998). DOI 9714612

18. Phadnis, A., Kumar, S., Srivastava, A.: Numerical investigation of thermal response of laser-irradiated biological tissue phantoms embedded with gold nanoshells. Journal of Thermal Biology 61, 16-28 (2016). DOI 10.1016/j.jtherbio.2016.08.002

19. Reynoso, F.J., Lee, C.d., Cheong, S.k., Cho, S.H.: Implementation of a multisource model for gold nanoparticlemediated plasmonic heating with near-infrared laser by the finite element method. Medical Physics 40(7), 073301 (2013). DOI 10.1118/1.4808361

20. Sapareto, S.A., Dewey, W.C.: Thermal dose determination in cancer therapy. International journal of radiation oncology, biology, physics 10(6), 787-800 (1984). DOI 10.1016/0360-3016(84)90379-1

21. Schweiger, M., Arridge, S.: The Toast++ software suite for forward and inverse modeling in optical tomography. Journal of biomedical optics 19(4), 040801 (2014). DOI 10.1117/1.JBO.19.4.040801

22. Silva, C.O., Rijo, P., Molpeceres, J., Ascensão, L., Roberto, A., Fernandes, A.S., Gomes, R., Pinto Coelho, J.M., Gabriel, A., Vieira, P., Reis, C.P.: Bioproduction of gold nanoparticles for photothermal therapy. Therapeutic delivery $\mathbf{7}(5), 287-304$ (2016). DOI 10.4155/tde2015-0011

23. Vera, J., Bayazitoglu, Y.: A note on laser penetration in nanoshell deposited tissue. International Journal of Heat 
and Mass Transfer 52(13-14), 3402-3406 (2009). DOI 10.1016/j.ijheatmasstransfer.2009.02.014

24. Welch, A.J., Van Gemert, M.J.C.: Optical-thermal response of laser-irradiated tissue, vol. 2. Springer, Dordrecht (2011). DOI 10.1007/978-90-481-8831-4

25. West, J.L., Hirsch, L.R., Stafford, R.J., Bankson, J.A., Sershen, S.R., Rivera, B., Price, R.E., Hazle, J.D., Halas, N.J., West, J.L.: Nanoshell-mediated near-infrared thermal therapy of tumors under magnetic resonance guidance. Proceedings of the National Academy of Sciences 100(23), 13549-13554 (2003). DOI $10.1073 /$ pnas.2232479100

26. Yao, R., Intes, X., Fang, Q.: Generalized mesh-based Monte Carlo for wide-field illumination and detection via mesh retessellation. Biomedical Optics Express 7(1), 171 (2016). DOI 10.1364/BOE.7.000171 